\title{
The Concept of a Scale in Accounting Measurement
}

\author{
SW Musvoto and DG Gouws
}

Department of Financial Management, University of Pretoria

Accepted November 2009

\begin{abstract}
This study compares the practices of accounting measurement with the principles of the representational theory of measurement to determine whether the attributes of accounting phenomena are measured on well-founded scales. The results of this study indicate that the concept of the representational scale is misapplied in the accounting discipline. The principles of representation measurement are hinged on the precise specification of how a scale of measurement is formed. Consequently, the findings suggest that accounting is not a measurement discipline.
\end{abstract}

Key words: Representational measurement, nominal scale, ordinal scale, interval scale, ratio scale

JEL M41

1

\section{Introduction}

The concept of a scale is an integral aspect of measurement. Every measurement process has to specify a scale of measurement (Ryan, Scapens \& Theobald, 2002). The specification of a scale of measurement is proof that the process is indeed one of measurement. Accounting is considered to be a measurement discipline (e.g., IASB, 2009; Wolk, Tearney \& Dodd, 2001). Consequently, one would expect to find specified scales of measurement in accounting. However, authors such as Staubus (2004), Ryan et al. (2002), Chambers (1997) and Willet (1987) have all pointed out that in the accounting discipline there is no specification of a scale of any kind that makes it possible to distinguish the extent to which every object in a specified class of accounting phenomena possesses a specified property. This contradicts the view that accounting is a measurement discipline. Narens (2002) also makes the point that a theory of measurement consists of a precise specification of how a scale is formed. The absence of specified scales of measurement in accounting therefore points to the absence of a theory of measurement in accounting.

According to Luce, Krantz, Suppes \& Tversk (1971), every process of measurement must have a theory of measurement. This means that every measurement discipline must have an underlying theory of measurement. The presence of a theory of measurement evidently guarantees the empirical validity of information produced by a process of measurement. Authors such as Staubus (1985) and Ijiri (1975) indicate a consensus that the accounting discipline has not succeeded in inferring a comprehensive and coherent theory of measurement from the observation of accounting measurement practices. Consequently, this suggests that researchers have not succeeded in specifying either how scales are formed in accounting measurement or whether the empirical validity of accounting information can be guaranteed.

The above discussion points out that accounting is considered to be a measurement discipline in the absence of evidence (scales) confirming that it is such a discipline. As a result, it is necessary to investigate whether this accounting perspective is a delusion. Accounting is classified as a social science (Ryan et al., 2002). This means that measurement in accounting is viewed from the perspective of social science. The theory that establishes measurement in social sciences is the representational theory of measurement (Luce et al., 1971, 1989, 1990). The concept of scale in accounting is thus discussed from the 
perspective of representational measurement theory.

The purpose of this study is to investigate whether numerical assignments in accounting are compatible with the concept of a scale in representational measurement. Such an investigation could find evidence supporting the viewpoint outlined in accounting literature that accounting information is empirically valid (is measurement information). Section 2 and its subsections present a discussion on the problem statement, the purpose of the research and the research methodology. The concept of a representational scale is discussed in section 3 , followed by a discussion of the significance of a scale in accounting in section 4. Sections $5,6,7$ and 8 discuss the properties of the individual scales of measurement and the way in which they are currently applied in the accounting discipline. The conclusions and recommendations are discussed in sections 9 and 10 respectively.

2

\section{Research}

\subsection{Statement of problem}

The problem addressed in this study concerns the reference to accounting as a measurement discipline in the absence of evidence (scales of measurement) supporting this viewpoint.

\subsection{Purpose of the research}

The purpose of this study is to find evidence to support the claim in accounting literature that accounting is a measurement discipline (i.e., accounting information is empirically valid), using the concept of a scale in representational measurement.

\subsection{Research methodology}

\subsubsection{Critical literature analysis}

The application of measurement theory to accounting is not a new idea. Vickrey (1970), noticing the absence of a measurement theory in accounting, questioned the status of accounting as a measurement discipline. He used the realist's theory of measurement to determine whether the necessary and sufficient conditions for classifying accounting as a measurement discipline exist in accounting. He concluded that accounting is not a measurement discipline, adding that it currently measures no property but the numerosity of monetary units. However, Vickrey's study (1970) overlooks the point that the realist's theory of measurement is not applicable to social sciences. He consequently evaluated the accounting concept of measurement from an erroneous perspective. According to Luce et al. (1971), in certain instances in the social sciences there is no available operation for facilitating the identification of extensive measurement, while in other instances the available operation leads either to trivial results or to a violation of the axioms. There are evidently structures with numerical representations that have no directly observable association operation, such as conjoint ones. This therefore betrays a lack of understanding of the principles of measurement on the part of accounting researchers.

Other authors, such as Staubus (1985, 2004), Chambers (1997), Abdel-Magid (1979) and Ijiri (1975), also highlight the lack of success in developing a theory of accounting measurement. This underlines the fact that numerical assignments in accounting are pretheoretic. All measurement magnitudes must be theoretically determined reflections of objectively existing entities (Decoene, Onghena \& Janssen, 1995). It can thus be argued that there is sufficient evidence to suggest that numerical assignments in accounting could be anything other than measurement magnitudes. To date, no major attempts have been made to resolve the measurement crises in accounting. Consequently, it is necessary to investigate the exact nature of numerical assignments in accounting.

\subsubsection{The representational theory of measurement}

The concept of a scale according to the representational theory of measurement is used in this study to investigate the exact nature of the numerical assignments in accounting. The representational theory of measurement was developed by Scott and Suppes in 1958. It is a 
theory of measurement comprising many related theories of measurement. This group of measurement theories requires in common a scale that can be defined by a set of structurepreserving mappings from a qualitative or empirically-based structure onto a structure from mathematics. The representational measurement theory offers an abstract theory of the kinds of well-behaved scales encountered in science. It places great emphasis on the uniqueness of representations. The list of scale types commonly found in science, and conceptualized by Stevens (1946, 1951), namely, ordinal, interval, ratio and nominal, has most empirical examples from representational measurement falling in this list. However, that is not to say that Stevens' (1951) list of scales is exhaustive. Narens (1981a, 1981b) shows that there are scales between the ratio and interval scales, but none of them has yet played a role in actual scientific measurement (Luce \& Suppes, 2001). For this reason, the discussion on scales in this study is limited to Stevens' (1951) list outlined above.

\section{3}

\section{The concept of a representational scale}

The concept of a scale is of fundamental importance in representational measurement. A scale is a rule used for the assignment of numerals to properties of objects or events (Stevens, 1951). This equates a scale to a specific method of measurement. Stevens (1951) also notes that measurement always occurs in a specific way, which means that every measurement process must have a rule of measurement. Consequently, it is clear that in a process of measurement the random assignment of numbers to objects is excluded.

Luce et al. (1971) argue that measurement can take place only if the rule that maps an empirical relational structure onto the numerical relational structure is specified. This indicates that the process of measurement takes place only in the presence of a standardized rule of measurement. Therefore, if the term "rule" in the above paragraph is taken to mean the presence of a scale in every measurement process, then it is reasonable to conclude that every process of measurement must have a scale of measurement. Similarly, Narens (2002:757) defines a scale as follows:

$S$ is said to be a representational scale if and only if there exists a qualitative structure $X$ and a mathematical representing structure $N$ for $X$ such that $S$ is a subset of one-to-one homomorphisms from $X$ to $N$.

This quotation points out that a scale of measurement can exist only when there is a qualitative structure that can be represented by a numerical relational structure. It is also clear that a scale is part of the homomorphisms that map a qualitative structure onto a numerical relational structure. A homomorphism is a function that maps an algebraic structure onto another in a way that preserves the properties of the algebraic structure being mapped (Bhattacharya, Jain \& Nagpaul, 1986). It is evident from this that a scale indicates the relationship that enables a qualitative structure to be mapped onto a numerical relational structure. It follows, therefore, that a scale explains how the properties of a qualitative structure are represented by an algebraic structure or a rule that explains the representation of an empirical relational structure by a numerical relational structure.

According to Luce et al. (1971) the scales of measurement are subject to arbitrary conventions. It can be argued from this that scales of measurement are socially constructed. It also suggests that each process of measurement has its own rules of measurement. As a result, there is the possibility of a proliferation of rules of measurement for a single process of measurement. Stevens (1951:1) refers to the possible proliferation of measurement rules when he states,

And the fact that numerals can be assigned under different rules leads to different kinds of scales and the different kinds of measurements.

These words underline the fact that there is no single method of measuring, but many. Thus, each type of scale distinguishes one form of measurement from another. These numerous methods of measurement lead to different kinds of scales. It can also be inferred that each 
frame of reference can have its own rules of measurement. In addition, it should be noted that the rules of measurement are not part of the phenomenon being measured. The empirical relational structure and its empirical properties are not a matter of convention. Luce et al. (1971) argue that the empirical relational structure and its empirical properties should be treated as a set of qualitative empirical laws. This means that the phenomenon being measured should be invariant under any set of measurement procedures. A set of measurement procedures does not change the underlying property it is measuring (e.g., a man's height is not changed in being measured by a metre rule).

Stevens (1951) characterized scales into four types, namely, nominal, ordinal interval and ratio scales. The type of scale achieved in measurement depends upon the character of the basic empirical operations performed on the property being measured. Ordinarily, these operations are limited by the peculiarities of the thing being scaled and by our choice of the concrete procedures. However, once selected, these procedures determine the type of scale that will eventuate (Stevens, 1951). The type of a scale indicates the level of measurement. Associated with each level of measurement is a set of mathematical operations that may be performed on a measure. Each level of measurement involves different properties (relations and operations) of the numbers or symbols that constitute the measurements.

The mathematical operations that may be performed on a measure without changing its meaning are termed permissible transformations on its scale. Permissible transformations on a scale preserve the relevant relationships of the measurement process (Luce et al., 1971). For example, changing the unit of measurement of distance (say, from inches to centimetres) multiplies the measurements by a constant factor. This multiplication does not alter the correspondence of the relationships "greater than" or the correspondence of addition and concatenation. Hence, it follows that the change of units is a permissible transformation with respect to these relationships.

The concept of a representational scale is inextricably linked to the uniqueness and the existence theorems of representational measurement. A number assigned to measure a property is unique once a unit of measurement has been chosen (Luce et al., 1990). This means that a scale of measurement makes a measure unique. It follows that the proof of the uniqueness theorem is equivalent to identifying all possible scales for the measurement of the elements of a given empirical relational system. It can therefore be concluded that the type of measurement can be known if, and only if, the scale of measurement is known.

\section{4}

\section{The significance of the concept of a representational scale in accounting}

The concept of a representational scale discussed in section 3 may be of fundamental significance to accounting. Mattessich (1964:63) identifies the nominal scale, ordinal scale, interval and ratio scales as the medium through which accounting information is communicated. According to Stevens (1951), the classification of scales into scale types is based on the amount of information about a property that is contained in a scale. This suggests that every measurement scheme should specify the type of scale used in order to indicate the amount of information contained by the measures it produces. It can thus be argued that a scale of measurement is an embodiment of the properties of the phenomenon being measured. It follows that, without the specification of a scale of measurement, it would be not possible to know what a particular numerical assignment represents. Therefore, if accounting were a measurement discipline, it should have been capable of specifying the rules of measurement employed in its measurement processes to give meaning to its numerical assignments. Failure to do so calls into question the status of accounting as a measurement discipline.

Measurement is about stating the relationship between the numerals and the objects. A rule of measurement states the relationship between the numerals and objects (Boyce, Meadow \& Kraft, 1994). This means that, if accounting has no specified rules of assigning numerals to objects, it implies that 
the relations between the numerals and objects are not known. If these relations are not known it would be difficult to determine the meaning of a measurement. It follows that the concept of a scale influences the meaningfulness of a measure. This is reflected by Chambers (1997:38):

In the third place, every measurement scheme requires the specification of the unit in the scale, and the conditions under which unit measurements shall be deemed to be of equal significance. In brief, this requires specification of the meaning of the "standard" unit. This is necessary since measurements may be taken in a variety of non-standard situations, such that the raw or crude measurements are not comparable or addable.

This indicates that a scale used in a process of measurement must be specified. The extract also points out that a scale gives empirical significance to measurements. Thus, a scale specifies the conditions under which a measurement has been made. It is clear from the above extract that the essence of meaningfulness is embodied in the description of the scale type and permissible statistics. This evidently highlights the fact that the statistics that can be performed on a measure lead to the formation of meaningful, or meaningless, statements based on measurements made on those scales. The meaning of a measure is therefore embodied in the description of the meaning of the standard unit. It can thus be concluded that, in the absence of specified scales, accounting measurements lack meaning and are incomparable.

Nevertheless, accounting information is considered comparable in the absence of specified scales of measurement. For example, IASB (2009) points out that users must be able to compare the financial statements of an entity through time in order to identify trends in its financial position and performance. This suggests that it is possible for users of accounting information to compare information from different entities in the absence of specified scales of measurement. The IASB (2009) points out that the measurement and display of the financial effect of like transactions and other events must be carried out in a consistent way throughout an entity and over time for that entity, and in a consistent way for different entities. This highlights the existence of a belief in accounting that measurement is possible in the absence of a specified scale of measurement. It can thus be concluded that the concept of a scale of measurement is not recognized in the accounting discipline.

The nature of accounting measurements demands that the scales of measurement should be specified before they are compared. This is because accounting measurements are dependent on the intuition of the accountant. As Mattessich (1964:79) states:

There neither exists at present the possibility to infer accounting values through "natural laws" (i.e., by fundamental measurement) nor through a combination of two or more fundamental measures that result in derived measurement. Most of the economic and accounting measures belong in the category of measurement by fiat, which is reflected in a certain definitional arbitrariness of our discipline.

This emphasizes that accounting measurements are dependent on the intuition of the accountant, as accounting is not a natural, but a social science. The use of the phrase "definitional arbitrariness of our discipline" implies that accounting definitions are not based on consistent rules or plans, but are instead dependent on the context in which they are used. It is clear that accounting measurements are socially constructed. Consequently, this suggests a need to specify clearly the nature of the social context of accounting measurements before they are evaluated. There could be a difference between the kinds of assigning of numbers arising from different procedures of measurement. Therefore, if a scale of measurement was not specified in a measurement discipline, it would not possible to tell whether there were any other numbers that could be assigned as measures of the same property. Such knowledge of other numbers that might be assigned is important in determining the uniqueness of a measure. The number assigned to measure a property of an object is unique 
once a unit has been assigned to it (Luce et al., 1971). The concept of a scale is thus also important for the quality of uniqueness of measures. A lack of specified scales of measurement implies that the uniqueness of numbers assigned to represent the properties of accounting objects cannot be determined.

The lack of specified scales of measurement has negative implications for the mathematical operations that could be carried out on accounting measurements. Chambers (1997) contends that the scales of measurements (or rather measurements taken in them) have different mathematical characteristics. He also suggests that the addition of measures and other forms of relations (inter alia, subtraction, multiplication or division) is common in accounting processes. As a result, it is necessary to consider the conditions under which addition (and other forms of relation) is mathematically permissible (e.g., the addition of different classes of assets). That is, the values of assets and liabilities are added in the balance sheet and in the income statement without first verifying whether these measurements have been made under the same scale of measurement.

The lack of specified scales causes inconsistencies in the classification of measures in accounting. Chambers (1997:39) notes the following on the classification of measures by the AAA's report (1971) on the foundations of accounting measures:

Among examples of primary measures are
counts of physical quantities, and prices of
non-monetary goods. In respect of prices, it
is said that they may be past, present or
future prices. No such stipulation is made in
respect of physical counts. Either, therefore,
counts and prices are not members of the
same class of measures (i.e. primary
measures), or both should be treated in the
same way (i.e., it should be allowed that
physical counts may be past, present or
future counts).

The above passage points out that accounting measures that differ are grouped in the same class, and that physical counts and prices are regarded as measures of the same property. It is also clear that there is no specified property represented by physical counts or by prices.
Furthermore, there is no specification of the scale of measurement that could be used to distinguish the extent to which physical counts and prices possess a particular property. Consequently, it is not clear whether physical counts and prices are measures of the same property. This leads to incorrect classification of measures. In this way, the lack of specified scales in accounting casts doubt on the current belief in the literature that accounting is a measurement discipline.

\section{5}

\section{The application of the concept of the nominal scale in accounting}

A nominal scale is the most basic scale of measurement. It is a simple classification or labelling system (Stevens, 1951). This suggests that only symbolic representation is necessary for measurement to occur under a nominal scale. Luce et al. (1971) note that the numbers in a nominal scale reflect the objects themselves, rather than their properties. For this reason, no mathematical operations may be performed on the numbers in a nominal scale. It follows that the only quantification is the number count of cases in each category (the frequency distribution). As a result the researcher is restricted to the use of the mode as the measure of central tendency (see Stevens, 1951). Hence, the nominal scale has limited arithmetic properties.

The nominal scale is commonly used in the accounting discipline. The activity of classification in accounting arises from the need for a tight net of a large number of concepts (Mattessich, 1964). Accounting phenomena are classified into five main classes of transactions relating to the statement of comprehensive income and the statement of financial position, namely, assets, liabilities, equity, income and expenses. Transactions classified under these headings are discrete and qualitative. They imply no order or any indication that they can be added. Nowhere in the accounting literature does it state or imply that mathematical operations of any kind can be performed on the classified transactions. However, there are instances in this literature that are unclear as to whether the nominal scale has been used to effect the mathematical 
operation of addition. For example, IAS 1 (2009) advocates that an entity present an analysis of expenses using a classification based on either the nature of expenses or their function within the entity, whichever provides reliable and more relevant information. This indicates that expenses will be classified in the financial statements according their nature and function. As has been outlined above (Stevens, 1951), seeing that the nominal scale is a simple classification system, it can be inferred that the classification of expenses based on their nature and function implies the use of a nominal scale. Furthermore, IAS 1 (2009) also indicates that, in analysing expenses, the first form of analysis is the nature of expenses method, and that expenses are aggregated in the statement of comprehensive income according to their nature (for example, depreciation, purchases of materials, transport costs, employee benefits and advertising costs), and are not reallocated among various functions within the entity. An analysis of this indicates that expenses may be classified according to their nature, but it does not mean that the monetary amounts of the individual expenses are representatives of an identical attribute of expenses under the same classification. A statement clearly indicating that the monetary amounts are representatives of identical attributes of expenses under the same classification is necessary, and the attribute must be specified. Nowhere in the IAS 1 (2009) is there any discussion of the attribute that the monetary amounts of expenses under the same classification represent in either general terms or in terms appropriate for their aggregation in the statement of comprehensive income. Willet (1987) notes that it is not known exactly what the monetary units represent in accounting. Researchers such as Vickrey (1970) and Ryan et al. (2002) have concluded that there is no property measured by the financial statements apart from the numerosity of monetary units. This leads to the conclusion that the nominal scale in this case is used to imply the operation of addition. In this case, it therefore follows that the nominal scale has been accorded qualities that are beyond identity and difference.

But in defence of the prescriptions of IAS 1 (2009), it may be contended that classification can be considered a form of measurement for a monothetic class. A monothetic class is one in which each member possesses all the properties that define the class. If addition is to be implied, then each member of the class must have all the properties that define that class. However, nowhere in IAS 1 (2009) is there any discussion of the properties that expenses falling under the same classification should have. This leads to the possibility that each member of the expenses falling under the same classification might possess a large number, but not necessarily all, of the properties that define that class. Consequently, classes in which expenses are classified in the financial statements might not be monothetic. One cannot thus easily imply addition without first verifying whether it is possible to add in a given set of circumstances. It can also be inferred that, in spite of the attempt by accounting researchers to use the nominal scale in the classification of accounting phenomena, they have not fully utilized the concept of this scale. This suggests that the concept of measurement might not be part of traditional accounting methodology; otherwise the accounting discipline would have ensured its proper development.

\section{6}

\section{The application of the concept of the ordinal scale in accounting}

The concept of the ordinal scale has its foundations in the concept of order. Order is the arrangement of things according to a particular sequence or method (Hawker, 2003). To be an order, the relationship must hold in only one direction when viewed relative to two members of the set (Stevens, 1951). That is, the relationship always looks the same irrespective of the angle from which it is viewed. This means that in all measurement instances involving the ordinal scale it is necessary to specify the property used to order empirical phenomena and the direction of the order.

The function of an ordinal scale is to assist in the determination of greater or lesser, such as the grades of wool or street numbers (Luce et al., 1971). It is an order of preference 
system. The use of the concept of the ordinal scale in the accounting discipline is evident, even though misapplied. For example, IAS 1 (2009) requires an entity to present assets and liabilities in order of liquidity only when a liquidity presentation provides information that is reliable and more relevant than a current/non-current presentation. This shows that the property "liquidity" is used to differentiate assets and liabilities in the statement of financial position into a hierarchy. The property of liquidity must therefore be unidirectional among current assets for an ordinal scale to exist. Moreover, IAS 1 (2009) also requires that entities such as financial institutions present assets and liabilities in increasing or decreasing order of liquidity to provide information that is reliable and more relevant than a current/non-current presentation, because the entity does not supply goods or services within a clearly identifiable operating cycle. This affirms the need to discriminate between assets and liabilities in the financial statements in order of liquidity. It can be inferred that the classification of assets or liabilities in increasing or decreasing order of liquidity implies the use of the ordinal scale. However, the numerals that specify the relative position of a current asset or a current liability in an ordered series defined by the property of liquidity are not provided. The absence of these numerals neglects the principles of the ordinal scale. Mattessich (1964:59) outlines the principles of the ordinal scale as follows:

The ordinal scale consists of classes that are characterized by numerals which are subject to order ranking in conformity with the numerals assigned. The numbers not only serve the mere purpose of designation, but also have a normative or preferential significance. It enforces such an order ranking and thus creates a hierarchy of classes. It is this order ranking which some scholars consider the decisive criterion of measurement.

This extract emphasizes that every ordinal scale should assign numbers to the property of the objects in a class in order for these objects to be ordered in accordance with how much of the property they possess. It can thus be inferred that the lack of numbers reflecting how much of the property of liquidity each component of current assets or liabilities possesses implies that the structure and calibration of the ordinal scale ordering current assets or liabilities according to the property of liquidity is not at present known in accounting. In section 3, it was noted that a measure is meaningful if the scale of measurement is known or if the transformations that leave its scale of measurement invariant are known. It would thus seem that it is currently impossible to establish any order preserving transformations that will leave the unknown scale of liquidity among current assets or liabilities invariant. Luce et al. (1971:38) make the point that any finite simple order can be represented by a finite set of real numbers, together with their natural ordering. The set of current assets or liabilities in the statement of financial position is finite. It is therefore necessary to specify the numbers that represent the liquidity of these assets or liabilities in the statement of financial position if an ordinal scale is to be established. A lack of numbers indicating liquidity also makes it difficult to prove the attributes of the relations that define order. Stevens (1951:14) identified these attributes as connectedness, asymmetry and transitivity. These characteristics are discussed below:

- Connectedness: The concept of connectedness is fundamental to defining order in a relation. A relation is connected when, given any two terms of its field, the relation holds between the first and the second or between the second and the first (Russell, 1920:33). That is, in a series of items, if any two are chosen there is a relation that holds between them. A relation has to be connected in order to arrange the elements of a set into a hierarchy. For example, a relation of "greater than" in a series of natural numbers implies that if any two different items are selected, one of them is greater than the other (Stevens, 1951:12). It is therefore necessary to know the connectedness of the relation of liquidity among current assets. If current assets are arranged in order of increasing liquidity in the statement of financial position, and two 
different current assets are chosen, then it should be shown that they are of differing liquidity.

- Asymmetry: An asymmetrical relation is a relation that holds in only one direction, for example, relationships such as "greater than" (if $y>x$ then $x$ is not greater than $y$ ), father of, or successor to (Stevens, 1951:13). It must be shown that order holds in one direction only. If the relation were to hold in more than one direction it would not be possible to establish the hierarchy of elements in a set. Therefore, if current assets are arranged in order of increasing liquidity in the statement of financial position, then it should mean that current assets that are high up in the series have liquidity greater than that of current assets that are lower in the series. For example, in the statement of financial position it should be empirically shown that cash is more liquid than inventories.

- Transitivity: The concept of the transitivity of relations is embedded in the concept of relations in abstract algebra. A transitive relation holds on more than two elements of a set without the elements having to be directly related. Bhattacharya et al. (1986:10) describe a transitive relation as follows: "Let $\mathbf{R}$ be a transitive relation on a set $\mathbf{X}$. $\mathbf{R}$ is said to be transitive if $\mathrm{x} \mathbf{R} \mathrm{y}$ and y $\mathbf{R} \mathrm{z}$ imply $\mathrm{x} \mathbf{R} \mathrm{z}$ for all $\mathrm{x}, \mathrm{y}, \mathrm{z}$ is an element of $\mathbf{X}$."

This definition suggests that if $\mathrm{x}$ is related to $\mathrm{y}$, and $y$ is related to $z$ in the same way it is related to $\mathrm{x}$, then $\mathrm{x}$ and $\mathrm{z}$ are related. Such a relationship is a transitive relationship. Transitivity is necessary in the determination of order in a relationship. The transitivity of a relationship should be empirically testable. If current assets are arranged in order of increasing liquidity in the statement of financial position, it should mean that liquidity as a relation among current assets is transitive. That is, if the liquidity of cash is greater than that of debtors, and the liquidity of debtors is greater than that of stock on hand, then it should be shown that the liquidity of cash is greater than that of stock if liquidity is to be transitive. From the discussion above it is clear that the lack of a scale of measurement that reflects the extent to which different current assets possess the property of liquidity means that the connectedness, asymmetry and transitivity of liquidity among current assets cannot be proved. Thus, an ordinal scale cannot exist among current assets and the concept has consequently been misapplied in accounting.

\section{7}

\section{The application of the concept of the interval scale in accounting}

The concept of the interval scale has its foundations in the equality of intervals. Stevens (1951) explains that the interval scale is quantitative in the ordinary sense of the word, and that all the usual statistical measures apply, unless they are the kinds that imply knowledge of a true zero point. It is evident from this that an interval scale has an arbitrary origin and that one may make all kinds of numerical statements about the interval scale apart from those that imply a true origin. Boyce et al. (1994) also refer to the arbitrary origin of the interval scale when they point out that all that is required in an interval scale is a point of origin and a unit of measurement. In accordance with the theory of the hierarchy of scales, the interval scale includes both the nominal scale and the ordinal scale (Stevens, 1951). In addition to its own extra properties, the interval scale includes order and classification. It is therefore clear that the interval scale includes classification, order and equality of intervals. The interval scale is concerned with the distance between or the closeness of two elements in a set (Boyce et al., 1994). This means that the value of the intervals between two elements in a set is the sum of the values of those intervals.

In the following discussion, applications of the interval scale by leading academics and professionals in the field of accounting are discussed. Wolk et al. (2001:9) illustrate the use of the interval scale in accounting as follows:

Thus, in accounting, both \$100,000 of current assets divided by \$50,000 of current liabilities and \$200,000 of current assets divided by \$100,000 of current liabilities 
indicate twice as much current assets as current liabilities.

This means that the monetary amounts that represent current assets can be divided by those that represent current liabilities to give the current ratio. This division indicates that a relationship is implied between the value of current assets and the value of current liabilities. It is also clear from this extract that current liabilities indicate amounts to be paid. The measure of the value of current assets represents what is available, in money or approximate money's worth, to pay off those liabilities. In order to cover the current liabilities, a measure of insolvency is sought. In order to make the assertion that there are twice as many current assets as current liabilities, one has to be sure that there is equality of ratios between the properties that are subject to division. Therefore, if there are twice as many current assets as current liabilities, there is the implication that the value of current assets is identical to the value of current liabilities. However, authors such as Stamp (1981), Tinker (1985) and McLean (2006) point out that value is a subjective concept that is not a property intrinsic to an accounting entity. It is evident that in current accounting, the empirical properties of value are not known, and that the measurable properties of value are unknown as well. For this reason, it is not true to imply the equality of ratios between the concept of value of an asset and the concept of value of a liability when the value is not known. Nor is it verifiable to assert the existence of a true zero point on an unknown scale of value measurement. That is to say, it is not true to assert that value is measurable on a ratio scale when the structure of value is currently not available for verification. Furthermore, as was pointed out above, an interval scale implies an equality of intervals between successive elements in a set, and it is also incorrect to imply the existence of equal intervals on an unknown scale of value measurement in both assets and liabilities. Thus, it can be concluded that it is not possible to imply the equality of intervals of an unknown variable.
8

\section{The application of the concept of the ratio scale in accounting}

The concept of the ratio scale has its foundations in the concept of the existence of four relations in an operation, namely, equality, order rank, equality of intervals, and equality of ratios (Stevens, 1951). The ratio scale is a combination of the nominal, interval and the ordinal scale, together with the equality of ratios. As a result, the ratio scale exhibits the properties of the nominal, interval and ordinal scales. Stevens (1951) also points out that all types of statistical operations are applicable to the ratio scales. It is thus clear that the ratio scale is quantitative in the ordinary sense of the word. The concept of the ratio scale in accounting is expressed in the use of financial ratios as indicators of performance. For example, referring to the quotation in section 4.6, Wolk et al. (2001:9) explain the use of the ratio scale in accounting as follows:

Using the ratio type scale of measurement in accounting is at least possible because the zero point implies nothingness in terms of dollar amounts. Thus, in accounting, both $\$ 100,000$ of current assets divided by $\$ 50,000$ of current liabilities and \$200,000 of current assets divided by $\$ 100,000$ of current liabilities indicate twice as much current assets as current liabilities. This is possible only because of the uniqueness of the zero point in accounting.

The excerpt highlights the fact that a ratio scale is created when accounting information is analysed with the use of ratios. The extract also points out that a ratio scale is created when the monetary amounts representing the value of current assets are divided by the monetary amounts representing the value of current liabilities to produce a current ratio. This division implies that the relationship between the number of monetary units assigned to represent the value of an asset and the asset's value is identical to the relationship between the numbers of monetary units assigned to indicate the value of a liability and the liability's value. But Ryan et al. (2002) note that there is no agreement relating the number of monetary units assigned to 
represent the value of a commodity and the value of a commodity. Thus the relationship between the number of monetary units assigned to represent the value of a commodity and the value of a commodity is ambiguous, and this relationship cannot be precisely specified. Indeed, it is clear that the relationship between current assets and current liabilities assumed in the creation of the current ratio cannot be empirically tested. It can therefore be concluded that the equality of ratios between current assets and current liabilities cannot be empirically tested.

According to Stevens (1951) measurement is possible because there is a kind of isomorphism between the empirical relations among objects and events and the numerical structures that represent them. This means that phenomena that cannot be precisely defined are not measurable. Since the relationship between the value of an asset or a liability and the number of monetary units used to represent it is not specified, the existence of such isomorphism cannot be verified. The measurement of intervals of monetary units is a ratio scale, but this does not mean that the monetary amount as a measure of the value of an asset or a liability is also a ratio scale. This is because value is subjective and as a result it is currently unknown whether value is measurable on a ratio scale. The ratio character of monetary unit measurement is based on the numerical representation of monetary intervals so that the value associated with the concatenation of adjacent intervals is the sum of values associated with those intervals. That is to say, monetary units can be represented on a number line. Furthermore, the concatenation of adjacent intervals of monetary units has, as far as is known, nothing empirically to do with the value of an asset or liability. Ryan et al. (2002) point out that monetary units have a standard scalar but there is no agreement relating them to a concept of value. If there is no reason to incorporate the monetary units into an empirical structure of the value of an asset or a liability, then there is nothing empirical about the representation of the value of an asset or a liability that limits which monotonic transformations of monetary units can be used as indices of value. This suggests that the belief that value can be represented numerically is pre-theoretic. There is an extensive theory for monetary unit measurements leading to ratio scale representations, indicating that monetary units can be represented by natural numbers. No comparable structure exists for the measurement of the values of assets or liabilities. A ratio scale exists for monetary units, but there is no independent theory for the measurement of the value of an asset or a liability, other than the pre-theoretic conjecture that the value of an asset or a liability is a monotonic function of monetary units. Moreover, there is no empirical relation between the notion of the value of an asset or a liability and the concatenations that pertain to the measurement of monetary units. It should also be pointed out that the division of current assets by current liabilities does not lead to a ratio scale, as the relationship between current assets and current liabilities is not specified. Narens (2002) believes it is necessary to specify the mathematical relations between objects before the assignment of numbers takes place. In this case, what is related between assets and liabilities is not known, as it is not specified in the accounting literature. Moreover, McLean (2006) considers value to be a subjective concept. It is evident from this that it is not possible to know the exact relationship between the amount of monetary units paid to acquire a commodity and its value. Therefore, current ratios as well as other accounting ratios are not based on the ratio scale. From this discussion it can thus be concluded that the concept of a ratio scale is misapplied in the discipline of accounting.

\section{9}

\section{Summary and Conclusions}

Although accounting is currently considered to be a measurement discipline, there is no evidence to support this conviction. The presence of a scale indicates that a discipline is a measurement discipline. However, this study has concluded that there are no specified scales of measurement in accounting. Rules for numerical assignments in accounting do not meet the criteria of a true scale of measurement. Consequently, this casts doubt on the veracity of the belief that accounting is 
a measurement discipline. A recapture of some of the main issues discussed indicates that:

- A scale is a rule of measurement that specifies the relationship between an empirical relational structure and a numerical relational structure. An example would be the use of a metre rule in the measurement of height. In abstract algebra a scale is referred to as a homomorphism.

- A nominal scale is a simple classification system. It is at the bottom of the hierarchy of scales. It has properties that reflect only the identity of the phenomena it is measuring. It is used in the accounting discipline to classify the elements of financial statements.

- An ordinal scale reflects the rank or order of the elements in a set. It discriminates between the elements in a set according to how much of the property an element in a set possesses. In the hierarchy of scales, it ranks higher than the nominal scale. It possesses the properties both of a nominal scale and of order.

- An interval scale reflects the equality of intervals between successive elements in a set. It is a higher-ranking scale than the nominal and the ordinal scales. It possesses the properties of a nominal scale, ordinal scale and the equality of intervals. This scale has an arbitrary starting point.

- A ratio scale reflects the equality of ratios among the elements in a set. It is a higherranking scale than the nominal, ordinal and the interval scales. It possesses the properties of the nominal, ordinal, and interval scales and the equality of ratios. This scale reflects the intrinsic properties of the object it is measuring. It has a true zero point.

Therefore, it can be concluded that, if accounting is to be considered a measurement discipline, its numerical assignments must be compatible with the concept of a scale in representational measurement.

\section{0}

\section{Recommendations}

Accounting can be considered a measurement discipline only if it complies with the principles of representational measurement. In particular, numerical assignments in accounting should specify the scale of measurement that distinguishes the extent to which elements in a particular class possesses a specified a property. Accounting should be redefined to incorporate the magnitudes that currently fall short of the qualities of measurements until such a time when it is fully developed as a measurement discipline. This recognizes the probable usefulness of numerical indices in accounting. Physical indices describe pre-theoretical variables as a useful initial step towards the development of a theory of measurement of such a variable, but this must not be confused with the fundamental measurement of the variable. Representational measurement theory helps clarify the status of a numerical assignment, whether indicant or measurement.

\section{References}

ABDEL-MAGID, M.F. 1979. Toward a better understanding of the role of measurement in accounting. The Accounting Review, April:346-357.

AMERICAN ACCOUNTING ASSOCIATION, 1971. Report of the committee on foundations of accounting measurement. The Accounting Review Supplement,

BHATTACHARYA, P.B., JAIN, S.K. \& NAGPAUL, S.R. 1986. Basic abstract algebra, Cambridge: Cambridge University Press.

BOYCE, B.R., MEADOW, C.T. \& KRAFT, D.H. 1994. Measurement in information science. Academic press limited.

CHAMBERS, R.J. 1997. Wanted: Foundations of accounting measurement, Abacus, (341):36-47.

DECOENE, S., ONGHENA, P. \& JANSSEN, R. 1995. Representationalism under attack. Journal of Mathematical Psychology, 39:234-242. 
HAWKER, S. 2003. The little Oxford dictionary, thesaurus and word power guide, Oxford University Press. IJIRI, Y. 1975. Theory of accounting measurement. American Accounting Association.

INTERNATIONAL ACCOUNTING STANDARDS BOARD (IASB), 2009, INTERNATIONAL

FINANCIAL REPORTING STANDARDS (IFRS), 2009: Including international accounting standards (IAS) and interpretations as at 1 January 2009.

LUCE, R.D.; KRANTZ, D.H.; SUPPES, P. \& TVERSK, A. 1971. Foundations of measurement, (Vol. 1) additive and polynomial representations, New York: Academic Press.

LUCE, R.D., KRANTZ, D.H., SUPPES, P. \& TVERSK, A. 1989. Foundations of measurement, (Vol. 2), Geometrical, threshold, and probabilistic representations, New York: Academic Press.

LUCE, R.D., KRANTZ, D.H., SUPPES, P. \& TVERSK, A. 1990. Foundations of measurement, (Vol. 3) Representations, axiomatization, and invariance, New York: Academic Press.

LUCE, R.D. \& SUPPES, P. 2001. Representational measurement theory. Available http:// media.wiley.com / product_data /except /87/ 04713788 0471378887.pdf.

MATTESSICH, R. 1964. Accounting and analytical methods, Homewood 3, Irwin

MCLEAN, R. 2006. New concepts in measuring value in Einstein (ed.) In the boardroom, John Wiley \& Sons, Inc.

NARENS, L. 1981a. A general theory of ratio scalability with remarks about the measurement -theoretic concept of meaningfulness. Theory and Decisions, 13:1-70.

NARENS, L. 1981b. On the scales of measurement. Journal of Mathematical Psychology, 24:249-275.

NARENS, L. 2002. A meaningful justification for the representational theory of measurement. Journal of Mathematical Psychology, 46:746-768.

RYAN, B., SCAPENS, R.W. \& THEOBALD, M. 2002. Research method and methodology in finance and accounting ( $2^{\text {nd }}$ ed.) Mitcham, Surrey: International, Padstow, Cornwall.

RUSSELL, B. 1920. Introduction to mathematical philosophy ( $2^{\text {nd }}$ ed.) New York: Macmillan.

STAUBUS, G.J. 1985. An induced theory of accounting measurement, The Accounting Review, January, 60(1):53-75.

STAUBUS, G.J. 2004. Two views of accounting measurement. Abacus, 40(3):265-279.

STAMP, E. 1981. Why can accounting not become a science like physics? Abacus, June:13-27.

STEVENS, S.S. 1946. On the theory of scales of measurement. Science, 103:667-680.

STEVENS, S.S. 1951. Mathematics, measurement and psychophysics in Handbook of experimental psychology, New York: Wiley.

SCOTT, D. \& SUPPES, P. 1958. Foundational aspects of theories of measurement. Journal of Symbolic Logic, 23:113-128.

TINKER, T. 1985. Paper prophets, A social critique of accounting. New York: Praeger.

VICKREY, D. W. 1970. Is accounting a measurement discipline?, Accounting Review, 45(4):731-742.

WILLET, R.J. 1987. An axiomatic theory of accounting measurement. Accounting and Business Research: $155-171$.

WOLK, H.I., TEARNEY, M.G. \& DODD, J.L. 2001. Accounting theory: A conceptual and institutional approach. South Western College Publishing. 